\title{
MicroRNA-30/Cx43 axis contributes to podocyte injury by regulating ER stress in diabetic nephropathy
}

\author{
Min $\mathrm{Li}^{1 \#}$, Weijie $\mathrm{Ni}^{2 \#}$, Mengyu Zhang ${ }^{1}$, Shusu Liu ${ }^{1}$, Mengting Chen ${ }^{2}$, Xiwei Hong ${ }^{2}$, Yubo $\mathrm{Ma}^{2}$, \\ Xinyang $\mathrm{Yu}^{2}$, Weilang $\mathrm{Wang}^{2}$, Min Yang ${ }^{1}$, Fei Hua ${ }^{3}$ \\ ${ }^{1}$ Department of Nephrology, the Third Affiliated Hospital of Soochow University, Changzhou, China; ${ }^{2}$ Southeast University School of Medicine, \\ Nanjing, China; ${ }^{3}$ Department of Endocrinology, the Third Affiliated Hospital of Soochow University, Changzhou, China \\ Contributions: (I) Conception and design: M Li, W Ni; (II) Administrative support: M Yang, F Hua; (III) Provision of study materials or patients: \\ M Zhang, M Chen; (IV) Collection and assembly of data: S Liu, X Hong, Y Ma; (V) Data analysis and interpretation: All authors; (VI) Manuscript \\ writing: All authors; (VII) Final approval of manuscript: All authors. \\ \#These authors contributed equally to this work. \\ Correspondence to: Prof. Min Yang, MD, PhD. Department of Nephrology, the Third Affiliated Hospital of Soochow University, Changzhou, China. \\ Email: yangmin1516@yeah.net; Prof. Fei Hua, MD, PhD. Department of Endocrinology, the Third Affiliated Hospital of Soochow University, \\ Changzhou, China. Email: czhuafei@vip.sina.com.
}

Background: The microRNA-30 family plays a critical role in the pathogenesis of podocyte injury. Cx43 plays an essential role in intercellular communication, which is essential for coordinated kidney function. This study was conducted to explore the function of microRNA-30s/Cx43 in podocyte injury in diabetic nephropathy (DN), both in vivo and in vitro.

Methods: SD rats were given streptozotocin (STZ) injections to induce DN. Podocytes were incubated in the medium in the presence or absence of high glucose (HG). The effects of the microRNA-30/Cx43 axis on DN and its underlying mechanisms were investigated by TUNEL assay, PAS, immunohistochemical staining, immunofluorescence staining, Western blot, RT-qPCR, RNA interference, and luciferase reporter assay. Podocytes were transfected with microRNA-30 family mimics, microRNA-30 family inhibitors, Cx43 siRNA, and negative controls to detect the effect of the microRNA-30/Cx43 axis. MicroRNA-30 family mimic AAVs, and microRNA-30 family inhibitor AAVs applied to regulate microRNA-30 family expression in the kidneys of the STZ-induced DN model rats to reveal the underlying mechanisms of the microRNA-30/Cx43 axis in DN.

Results: MicroRNA-30 family member expression was downregulated in HG-treated podocytes and the glomeruli of STZ-induced DN rats. Luciferase reporter assays confirmed $\mathrm{Cx} 43$ is a directed target of microRNA-30s. The overexpression of microRNA-30 family members attenuated the HG-induced podocyte injury and protected against podocyte apoptosis and endoplasmic reticulum stress (ERS) both in vivo and in vitro. Also, silencing $\mathrm{Cx} 43$ expression eased podocyte apoptosis, injury, and ERS induced by a HG+microRNA-30 family inhibitor. Double-immunofluorescence staining assays proved the co-localization of caspase 12 and $\mathrm{Cx} 43$.

Conclusions: The overexpression of microRNA-30 family members prevents HG-induced podocyte injury and attenuates ERS by modulating $\mathrm{Cx} 43$ expression. The microRNA-30/Cx43/ERS axis might be a potential therapeutic target to treat DN.

Keywords: MicroRNA-30s; Cx43; endoplasmic reticulum stress (ERS); podocyte; diabetic nephrology

Submitted Aug 06, 2020. Accepted for publication Dec 01, 2020.

doi: 10.21037/atm-20-6989

View this article at: http://dx.doi.org/10.21037/atm-20-6989 


\section{Introduction}

Diabetic nephropathy (DN) is one of the most severe complications of diabetes mellitus and is the most common cause of end-stage renal disease worldwide (1). However, the pathogenesis of DN is not fully understood (2). As terminally differentiated cells, glomerular podocytes play essential roles in supporting the structure and function of the renal filtration barrier (3). Growing evidence shows the depletion and loss of glomerular podocytes are the earliest signs of DN (4). Thus, it is crucial to elucidate further the pathogenesis of podocyte injury for the early prevention and treatment of $\mathrm{DN}$.

The microRNA-30 family is evolutionarily conserved and comprises five members, including microRNA-30a, microRNA-30b, microRNA-30c, microRNA-30d, and microRNA-30e (5). It has been reported microRNA-30s take part in several cellular processes, including autophagy, DNA damage, and apoptosis (6). Compared with other glomerular cell types, podocytes abundantly express microRNA-30 family members (7). The pathogenesis of microRNA30 s involved in podocyte injury contain the regulation of calcium/calcineurin signaling (8), the regulation of Notch1 and p53 signaling (5) and the regulation of high glucose (HG)-induced p38 MAPK-dependent pathway by targeting the expression of metadherin (9). MicroRNA-30 has been also reported to inhibit epithelial-mesenchymal transition of podocyte cells (10). Moreover, microRNA-30 has been reported to suppress inflammatory factor-induced endothelial cell injury by targeting TCF21-MAPK/p38 signaling pathway (11), to regulate angiogenesis by suppress the expression of delta-like 4, a membrane-bound ligand belonging to the Notch signaling family (12) and to inhibit neointimal hyperplasia by targeting $\mathrm{Ca}^{2+} /$ calmodulindependent protein kinase II $\delta$ (13), which may be involved in the progression of atherosclerosis. Despite the above studies, the exact functions of microRNA-30s remain unknown concerning DN.

Gap junctions (GJs) are clusters of intercellular channels found at the plasma membrane that mediate direct cell-tocell communication and play critical roles in cell death, cell proliferation, and tissue development (14). Connexin-43 (Cx43), encoded by the GJA1 gene on chromosome 6, is a part of GJs and is the primary connexin member in podocytes (15). Podocyte $\mathrm{Cx} 43$ channels are essential for coordinated kidney function. Increasing evidence shows that the upregulation of $\mathrm{Cx} 43$ expression is a marker of injury in podocytes $(16,17)$. It has been reported the transfection of $\mathrm{Cx} 43$ in the LLC-PK1 cell line promotes endoplasmic reticulum stress (ERS)-induced apoptosis (18). Despite these studies, the functions of $\mathrm{Cx} 43$ in podocyte injury and the relationship between Cx43 and ERS are still poorly understood, particularly concerning kidney disease.

The endoplasmic reticulum (ER) is a critical organelle that mediates cell death. Briefly, many conditions lead to the accumulation of unfolded proteins within the ER, and ERS induces cells to correct the environment within the ER. However, if the stimuli are too severe, and homeostasis cannot be restored, ERS activates apoptotic signaling (19). Caspase12 plays a crucial role in ERS-induced apoptosis (20). It has been reported caspase12-deficient mice are resistant to ERS-induced apoptosis, and the activation of caspase12 is mediated only by ERS (21). Earlier studies have shown ERS-induced apoptosis occurs in DN (21). However, the precise mechanisms involved in ERS remain unknown.

To our knowledge, there have been no report about the contribution of microRNA-30/Cx43 axis to podocyte injury in DN. In this study, we used HG-treated podocytes and streptozotocin (STZ)-induced diabetic rats as experimental models of $\mathrm{DN}$ to investigate the mechanism of the microRNA-30 family in podocyte injury. We found $\mathrm{Cx} 43$ is a directed target of microRNA-30s and that the overexpression of microRNA-30s eases ERS by inhibiting $\mathrm{Cx} 43$ expression. Thus, this study reveals the underlying mechanism of microRNA-30s in podocyte injury, which supplies a new target for DN treatment. We present the following article in accordance with the ARRIVE reporting checklist (available at http://dx.doi.org/10.21037/atm-206989).

\section{Methods}

\section{Animal models}

All animal experiments were performed in accordance with the guidelines for animal care and approved by the Institutional Animal Care and Use Committee at Soochow University, China (No. 2020-013). The SD rats weighing 200-250 g (8-to-10 weeks of age) received an STZ injection $(60 \mathrm{mg} / \mathrm{kg}$ solution in citrate buffer, via a single intraperitoneal injection) after being fasted overnight. A blood glucose level of $\geq 350 \mathrm{mg} / \mathrm{dL}$ was indicative of diabetes. The microRNA-30 mimic AAVs, and the microRNA-30 inhibitor AAVs were constructed by HanBio (www.hanbio.net). The rats were randomly chosen to receive a single-bolus tail vein injection of either 
microRNA-30 mimic AAV or the microRNA-30 inhibitor $\mathrm{AAV}$ at $1 \times 10^{11}$ viral genomes per animal.

\section{Podocyte culture and treatment}

Dr. Yong Gu provided the human podocyte cell line (Department of Nephrology, Huashan Hospital of Fudan University, Shanghai, China), and the cells were cultured as previously described (5). Podocytes were moved to $37^{\circ} \mathrm{C}$ for differentiation and cultured without interferon- $\gamma$. The podocytes were grouped and transfected with microRNA-30 mimics, microRNA-30 inhibitors, Cx43 siRNA, and negative controls (Invitrogen, Carlsbad, CA). The transfected cells were growth-arrested by incubation with serum-free medium overnight before incubation with $5.5 \mathrm{mM}$ glucose (Invitrogen, Carlsbad, CA) or $25 \mathrm{mM}$ glucose (HG) for 48 hours for further experimental analysis.

\section{Periodic acid-Schiff staining and glomerular sclerosis score}

Kidney sections $(3 \mu \mathrm{m})$ were stained with periodic acidSchiff (PAS). The severity of injury for each glomerulus was scored from 0 to 4 : 0 , no lesions; $1,<25 \%$ involvement of the glomerulus; 2, 25-50\% involvement; 3, 50-75\% involvement; and 4, >75\% involvement. Fifty glomeruli were analyzed per kidney section. A glomerular sclerosis score (GSS) for each animal was calculated by multiplying each severity score [0-4] by the percentage of glomeruli displaying that injury and summing these scores. The above histological analysis was performed blinded to avoid bias.

\section{Apoptosis studies}

The cytosolic DNA-histone complexes generated during apoptotic DNA fragmentation in treated podocytes were tested with a cell death detection ELISA kit (Cell Death Detection ELISA PLUS; Roche Applied Science, Indianapolis, IN, USA) and performed by following the manufacturer's protocol.

\section{Western blot and antibodies}

Podocytes harvested from plates and sieved glomeruli were lysed in SDS sample buffer containing $150 \mathrm{mM} \mathrm{NaCl}, 0.1 \%$ Triton X-100, 0.5\% deoxycholate, 0.1\% sodium dodecyl sulfate (SDS), $50 \mathrm{mM}$ Tris- $\mathrm{HCl}$ (pH 7.0), and $1 \mathrm{mM}$ ethylenediaminetetraacetic acid (EDTA). The detection of protein expression by Western blot was conducted according to established protocols. The primary antibodies used were: Cx43 (1:200), caspase12 (1:200), podocin (1:200), and GAPDH (1:1,000). Densitometric analysis was performed using Quantity One Software (Bio-Rad). Antibodies against $\mathrm{Cx} 43$ and caspase 12 were bought from Cell Signaling Technology (Beverly, MA). Anti-podocin antibodies were retrieved from Abcam (Cambridge, MA).

\section{RNA's extraction and quantitative real-time PCR}

Total RNA from podocytes and kidney tissues was extracted with an amirVana miRNA Extraction kit (Jiancheng, Nanjing, China). The following PCR parameters were used for real-time qPCR: $94^{\circ} \mathrm{C}$ for 2 minutes and 45 cycles of $95^{\circ} \mathrm{C}$ for 15 seconds and $60^{\circ} \mathrm{C}$ for 60 seconds. The U6 small nuclear RNA was used as the internal control for individual miRNA level analysis in the cells and tissues. The expression of miRNA was calculated as the difference between the threshold values of the two genes $(\Delta \Delta \mathrm{CT})$.

\section{IHC and IF}

We prepared $3-\mu \mathrm{m}$ sections of the paraffin-embedded kidney tissues and subjected these sections to immunohistochemistry (IHC) using peroxidase-conjugated antibodies and DAB for visualization. The sections were incubated with Cx43 (Cell Signaling Technology; Beverly, MA). Integrated optical density (IOD) was used to stand for the relative amount of positive staining. For immunofluorescence (IF) staining, 5- $\mu$ m sections of frozen tissue samples were blocked with BSA and incubated in primary antibodies against caspase-12 (Abcam; Cambridge, MA) and Cx43 (Abcam; Cambridge, MA).

\section{Luciferase reporter assay}

The 3'-UTR of $\mathrm{Cx} 43$, with wild-type or mutated (Mut) binding sites for microRNA-30s, was amplified and cloned into the pGL3 vector to generate the plasmid pGL3-wtCx43-3'-UTR or pGL3-mut-Cx43-3'-UTR, respectively. For the luciferase reporter assay, HEK-293 cells were cotransfected with the luciferase reporter vectors and microRNA-30 mimics, microRNA-30 inhibitors, or the corresponding negative control using Lipofectamine 2000 reagent. The pRL-TK plasmid (Promega, Madison, USA) was used as a normalization control. After 48 hours of incubation, a Luciferase Reporter Assay kit (Promega) was used to detect luciferase activity according to the 
manufacturer's instructions.

\section{Statistical analysis}

Data are expressed as the mean $\pm \mathrm{SD}$. Comparisons between groups were performed with one-way ANOVA followed by Dunnett's multiple comparison tests or Student's $t$-test. $\mathrm{P}<0.05$ was considered statistically significant.

\section{Results}

\section{Cx43 is a microRNA-30 family target gene}

As presented in Figure 1A, we found that the expression of all five members of the microRNA-30 family was downregulated by HG administration. To explore the possible molecular basis by which microRNA-30s regulate the DN process, we searched for potential microRNA-30 family targets using TargetScan and identified $\mathrm{Cx} 43$ as a potential microRNA-30 family target. As shown in Figure 1B, microRNA-30s contain binding sites complementary to the 3'-UTR of $\mathrm{Cx} 43$. Luciferase activity determination also revealed the functional interaction between microRNA-30 members and $\mathrm{Cx} 43$ (Figure 1C). To further investigate the actual effect of microRNA-30s on $\mathrm{Cx} 43$ expression, podocytes were transfected with microRNA-30 family mimics or inhibitors. Quantitative PCR, IF staining, and Western blotting revealed that HG treatment induced an increase in $\mathrm{Cx} 43$ expression in podocytes, while the introduction of microRNA-30 family abated this effect mimics substantially. Compared with the HG group, the microRNA-30 family inhibitor-treated group had a significantly upregulated expression of $\mathrm{Cx} 43$ (Figure 1D,E,F,G). Collectively, all the above results show $\mathrm{Cx} 43$ is a direct target of the microRNA-30 family.

\section{MicroRNA-30s attenuate apoptosis, injury, and ERS in podocytes in vitro}

As shown in Figure 2, we confirmed that compared with that in the HG group, the protein expression of caspase12, a marker of ERS, and the level of apoptosis in the microRNA-30 family mimic transfection group was markedly reduced. However, the protein expression of podocin was significantly increased, which means microRNA-30 mimics can protect podocytes against HGinduced injury. The above results were reversed in the microRNA-30 family inhibitor transfection group.
Inbibition of Cx43 attenuates podocyte apoptosis, injury, and ERS in vitro

As shown in Figure 3, compared with the control group, the apoptosis, and the protein expression of $\mathrm{Cx} 43$ and caspase 12 in the HG group increased. However, the protein expression of podocin was decreased. The HG-induced apoptosis, injury, and ERS of podocytes were reduced by Cx43 siRNA intervention but upregulated by microRNA-30 family inhibitor intervention. Compared with the HG + microRNA-30 inhibitors group, the HG + microRNA-30 inhibitors + $\mathrm{Cx} 43$ siRNA group had lower levels of apoptosis, Cx43 expression, and caspase 12 expression and higher levels of podocin expression.

\section{Downregulation of microRNA-30s enbances STZ-induced Cx43 expression in DN rats}

As shown in Figure 4, we performed qPCR to detect the expression of microRNA-30 family members in STZinduced DN rats and found STZ downregulated the expression of all members of the microRNA-30 family. The microRNA-30mimicinjection increased the expression of microRNA-30 family members, while the injection of microRNA-30 inhibitors had the opposite effect. Compared with that in the control group, the protein expression of $\mathrm{Cx} 43$ in the STZ group was significantly increased, and could be reduced by microRNA-30 mimics but was further increased by microRNA-30 inhibitors.

\section{Downregulation of microRNA-30 family expression enhances STZ-induced kidney injury in DN rats}

To test the effect of the microRNA-30 family on the renal injury, STZ-induced DN rats were intravenously injected with microRNA-30 family mimics or inhibitors. As shown in Figure 5, the glomerular sclerosis scores revealed by PAS staining were markedly increased in STZ-induced DN rats compared to control rats and were notably abrogated by microRNA-30 mimic family injection. Then we found that microRNA-30 family inhibitor injection increased the glomerular sclerosis scores. As displayed in Figure 5, the expression of podocin was downregulated by STZ and could be rescued by microRNA-30 family mimics. Yet, microRNA-30 family inhibitors reduced the podocin expression. These data show the inhibition of microRNA-30 family member expression enhances STZ-induced kidney injury in $\mathrm{DN}$ rats. 
A

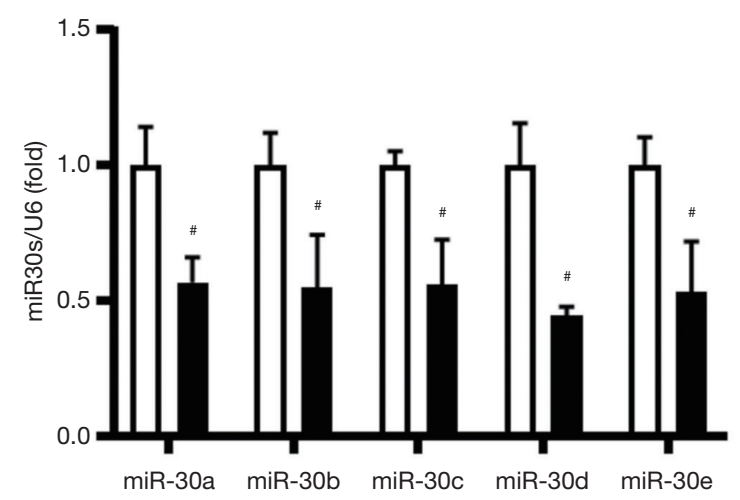

Control

HG
B Position 971-978 of Cx43 3`UTR

hsa-miR-30a-5p hsa-miR-30b-5p hsa-miR-30c-5p hsa-miR-30d-5p hsa-miR-30e-5p
5'......UGUGGGCCAAUAUGGUGUUUACA......

| | | | | | | |

GAAGGUCAGCUCCUACAAAUGU UCGACUCACAUCCUACAAAUGU CGACUCUCACAUCCUACAAAUGU GAAGGUCAGCCCCUACAAAUGU GAAGGUCAGUUCCUACAAAUGU

\section{C

$$
\text { C }
$$

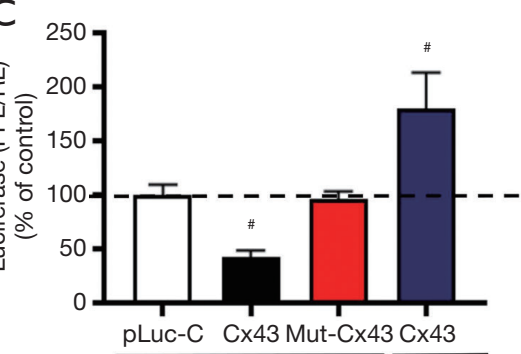

F

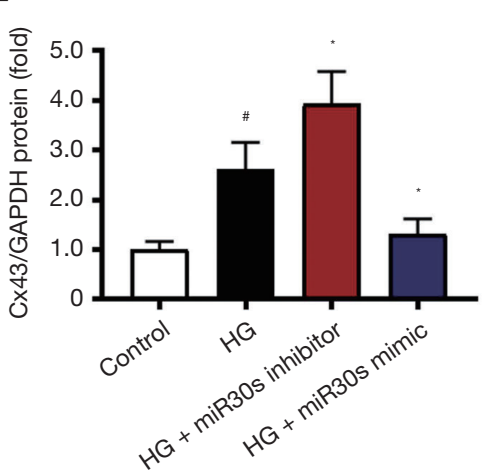

D
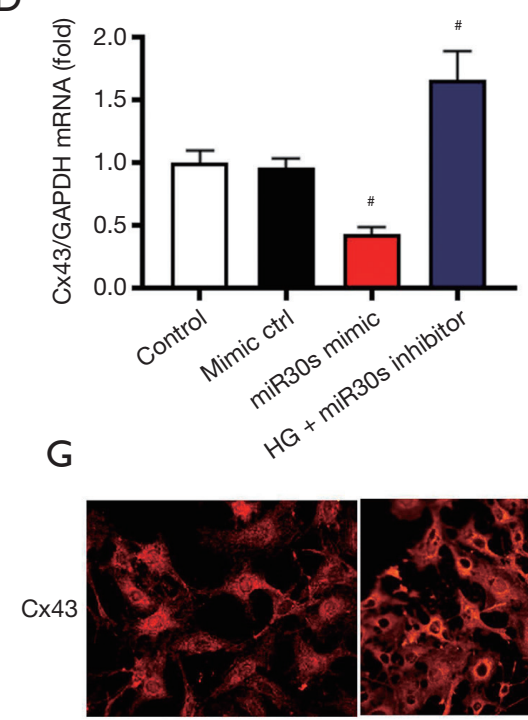

Control
E

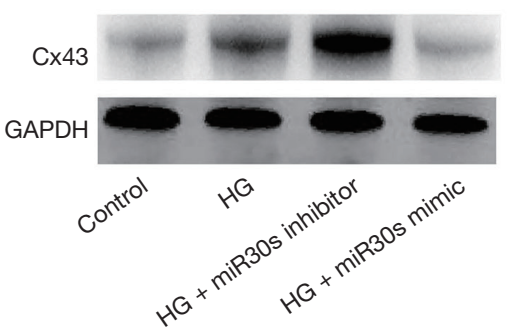

Figure $1 \mathrm{Cx} 43$ is a direct target of microRNA-30s. (A) Changes in the expression of microRNA-30 family members in podocytes treated with vehicle or HG. " , $\mathrm{P}<0.05$ vs. the control group. (B) The putative microRNA-30 family binding sites in the 3 '-UTR of Cx 43 predicted by TargetScan. (C) Luciferase activity assays in podocytes transfected with microRNA-30 family mimics or inhibitors for 48 hours. ", $\mathrm{P}<0.05$ vs. microRNA-30 mimics + pLuc-C. *, P<0.05 vs. microRNA-30 mimics + Cx43. (D) RT-qPCR analyses of the mRNA expression of Cx43 in podocytes treated with control, mimic control, microRNA-30 family mimics, or microRNA-30 family inhibitors + HG. ${ }^{*}, \mathrm{P}<0.05$ vs. the control group. (E) Western blotting analyses of the protein expression of Cx43 in podocytes treated with vehicle, HG, microRNA-30 family mimics + HG, or microRNA-30 family inhibitors + HG. (F) Quantitative analyses of the relative abundance of Cx43 protein expression expressed as the mean \pm SEM of three experiments. ${ }^{\#}, \mathrm{P}<0.05$ vs. the control group. ${ }^{*}, \mathrm{P}<0.05$ vs. the HG group. (G) IF staining for $\mathrm{Cx} 43$ in podocytes treated with vehicle, HG, microRNA-30 family mimics + HG, or microRNA-30 family inhibitors + HG (original magnification, ×400). HG, high glucose; IF, immunofluorescence. 

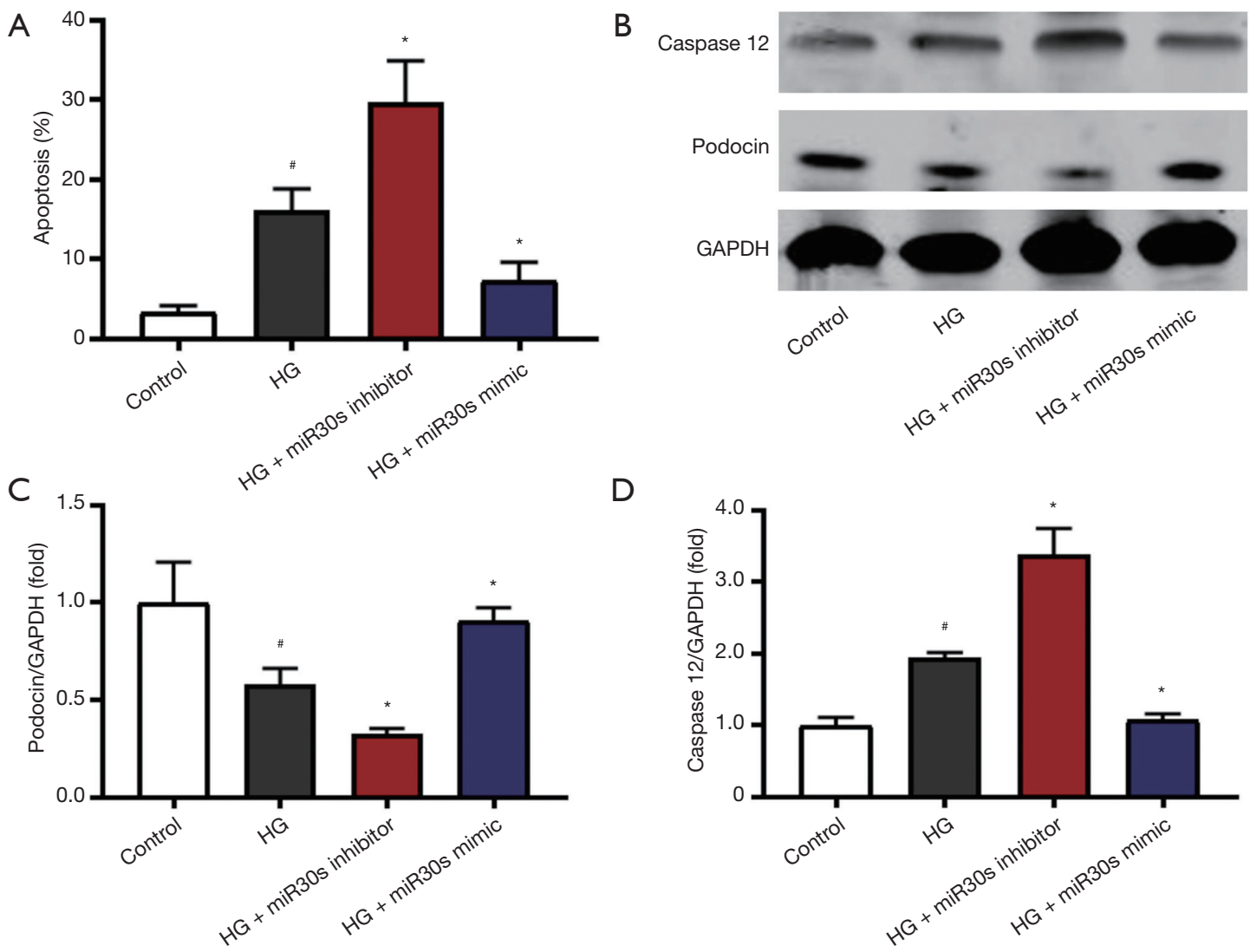

Figure 2 MicroRNA-30s alleviatepodocyte apoptosis, injury, and ERS in vitro. (A) Analyses of apoptosis in podocytes treated with vehicle, HG, microRNA-30 family mimics + HG, or microRNA-30 family inhibitors + HG. (B) Western blotting analyses of the protein expression of podocin and caspase12 in podocytes treated with vehicle, HG, microRNA-30 family mimics + HG, or microRNA-30 family inhibitors + HG. (C) Quantitative analyses of the relative abundance of podocin protein expression expressed as the mean \pm SEM of three experiments. (D) Quantitative analyses of the relative abundance of caspase12 protein expression expressed as the mean $\pm \mathrm{SEM}$ of three experiments. ${ }^{\#}$, $\mathrm{P}<0.05$ $v s$. the control group. *, $\mathrm{P}<0.05$ vs. the HG group. HG, high glucose; ERS, endoplasmic reticulum stress.

\section{Downregulation of microRNA-30 family expression enhances STZ-induced ERS in DN rats}

Western blotting and double-immunofluorescence staining assays were used to examine the expression of caspase 12 and $\mathrm{Cx} 43$ in kidney tissues. Compared with those in the control group, the levels of caspase 12 and $\mathrm{Cx} 43$ in the STZ group were markedly increased. The levels of caspase 12 and $\mathrm{Cx} 43$ were increased by microRNA-30 family inhibitors but were inhibited by microRNA-30 family mimics (Figure 6A,B). Also, as shown in Figure $6 C$, caspase 12 and $\mathrm{Cx} 43$ were colocalized in the podocytes of STZ-induced DN rats.

\section{Discussion}

In the present study, we verified that the expression of microRNA-30 family members was markedly downregulated in STZ-induced DN rats and HGstimulated podocytes. We found $\mathrm{Cx} 43$ might be a target of the microRNA-30 family and that the overexpression of microRNA-30s protects podocytes against injury. Our data show the overexpression of the microRNA-30 family exerts anti-ERS effects by inhibiting $\mathrm{Cx} 43$ expression. The microRNA-30/Cx43/ERS axis might be a new successful therapeutic target for $\mathrm{DN}$.

$\mathrm{DN}$ is a severe microvascular complication of diabetes with multiple pathogenic factors and limited treatment options (22). Earlier studies have proved podocyte apoptosis is associated with the pathological mechanisms of DN (23). Although multiple signaling pathways take part in podocyte apoptosis, the mechanism underlying podocyte apoptosis is 

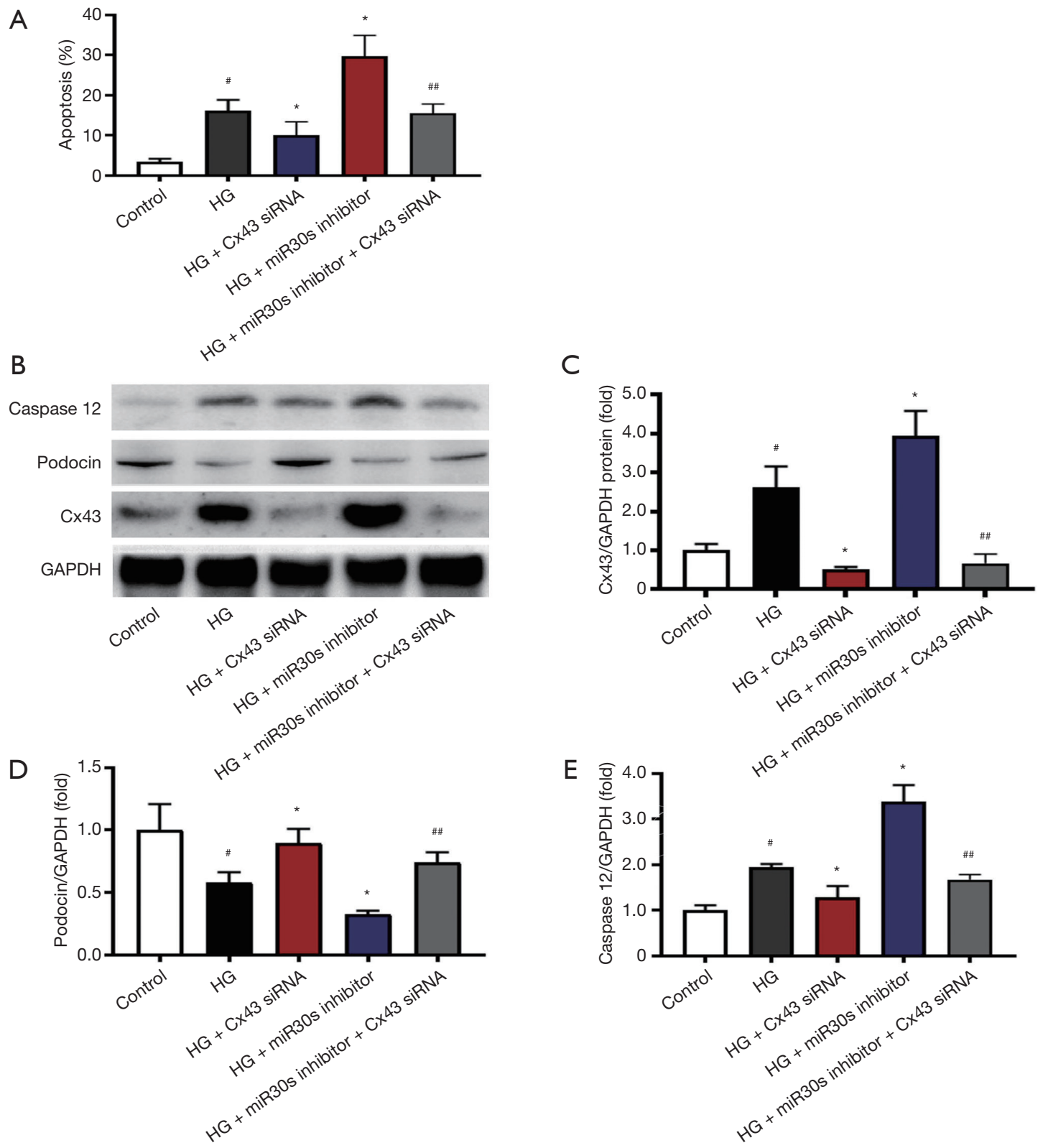

Figure 3 Inhibition of $\mathrm{Cx} 43$ reduces podocyte apoptosis, injury, and ERS induced by HG or HG + microRNA-30 family inhibitors in vitro. Podocytes were distributed into five groups: control, HG, HG + Cx43 siRNA, HG + microRNA-30 family inhibitors, and HG + microRNA-30 family inhibitors + Cx43 siRNA. (A) Analyses of podocyte apoptosis among the five groups. (B) Western blot analyses of the protein expression of caspase12, Cx43, and podocin among the five groups. $(\mathrm{C}, \mathrm{D}, \mathrm{E})$ The quantitative ratios are shown as the relative optical densities of the bands that are normalized to the expression of GAPDH. The data are representative of three similar experiments and quantified as the mean \pm SEM. ${ }^{*}, \mathrm{P}<0.05$ vs. the control group. ${ }^{*}, \mathrm{P}<0.05$ vs. the HG group. ${ }^{\#}, \mathrm{P}<0.05$ vs. HG + microRNA-30 family inhibitors. HG, high glucose; ERS, endoplasmic reticulum stress.

still incompletely understood. Therefore, it is of considerable significance to explore the pathogenic mechanism of podocyte injury in DN. STZ-induced DN rat models have been widely used to investigate the pathogenesis of DN (24), and hyperglycemia is a major pathogenic factor in renal injury (25). Therefore, STZ-induced DN rat models and HG-stimulated podocytes were applied as in vivo and in vitro models in this research. 


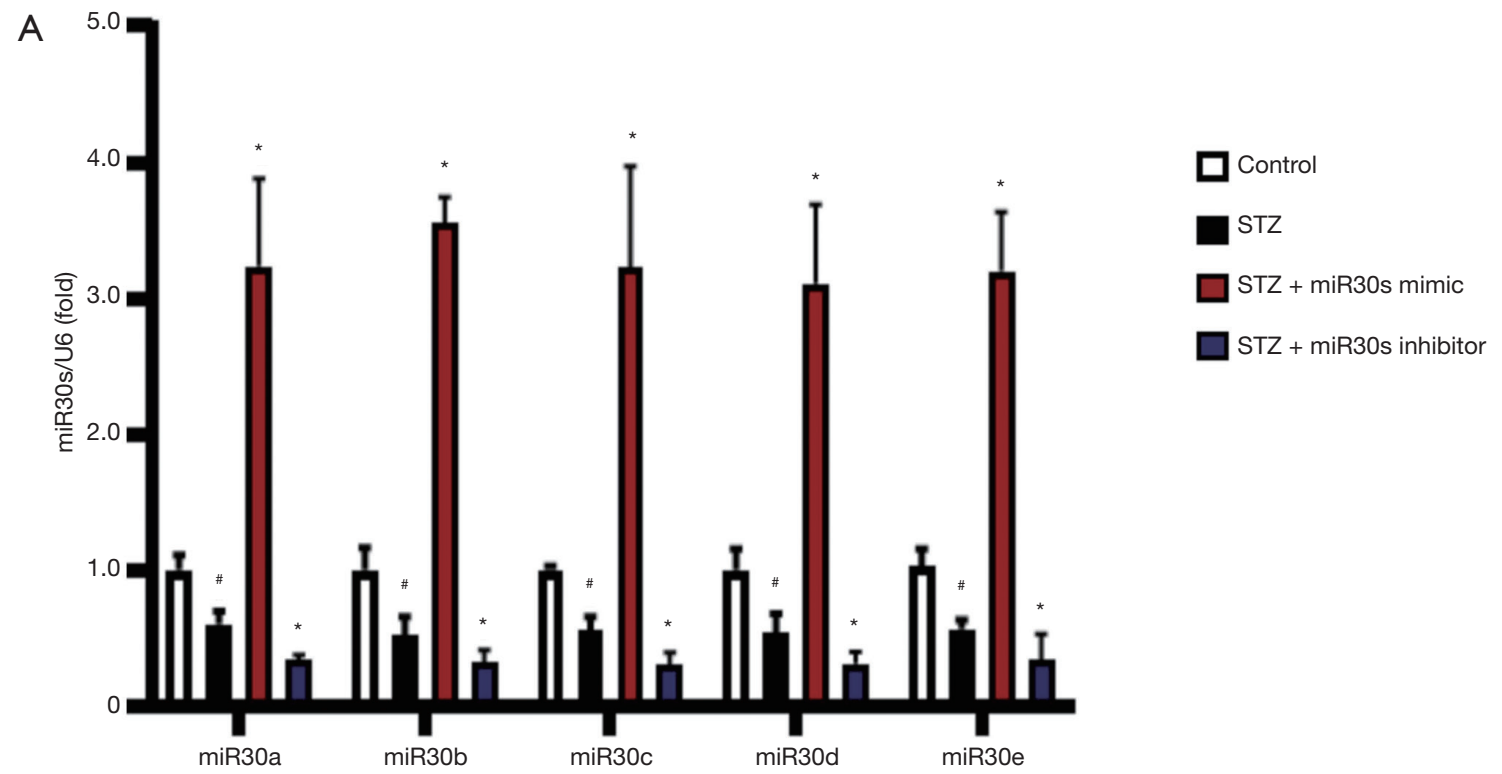

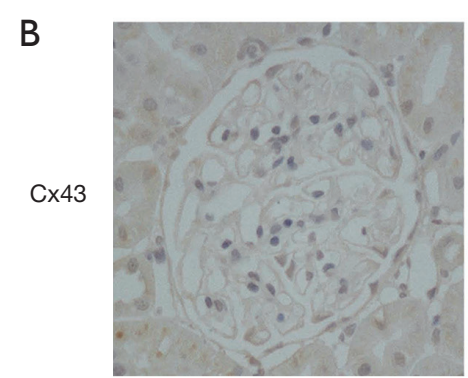

Control

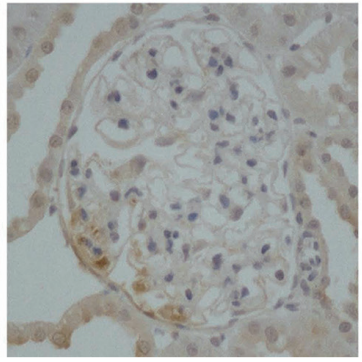

STZ

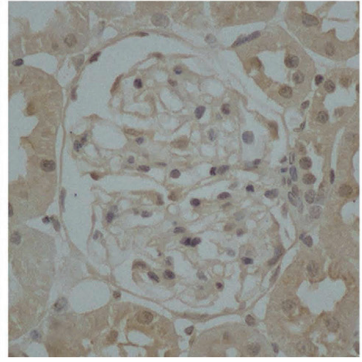

$\mathrm{STZ}+\mathrm{miR} 30 \mathrm{~s}$ mimic

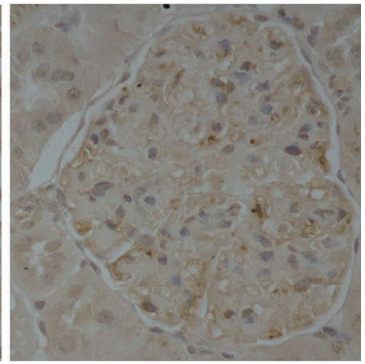

$\mathrm{STZ}+\mathrm{miR} 30 \mathrm{~s}$ inhibitor

C

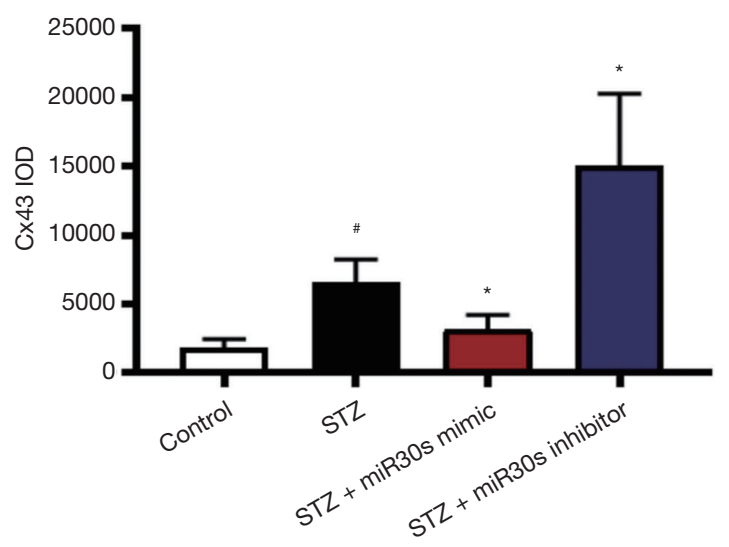

Figure 4 MicroRNA-30s modulate Cx43 expression in STZ-induced DN rats. Rats were divided into four groups: control, STZ, STZ + microRNA-30 family mimics, and STZ + microRNA-30 family inhibitors. (A) RT-qPCR analyses of microRNA-30s among the four groups. (B) IHC analyses of $\mathrm{Cx} 43$ protein expression among the four groups ( $\times 40)$. Representative images from 4 mice in each group are shown. (C) Quantitative analyses of the IOD of $\mathrm{Cx} 43$ are shown in the bar graph, and data are expressed as the mean \pm SEM of three experiments. \#, $\mathrm{P}<0.05$ vs. the control group. * $\mathrm{P}<0.05$ vs. the STZ group. STZ, streptozotocin; DN, diabetic nephropathy; IHC, immunohistochemistry. 


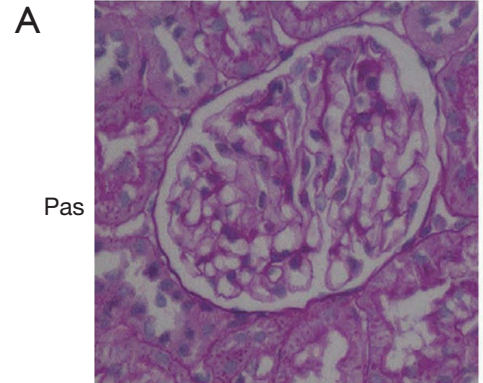

Control

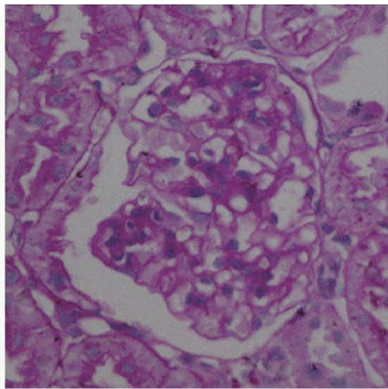

STZ

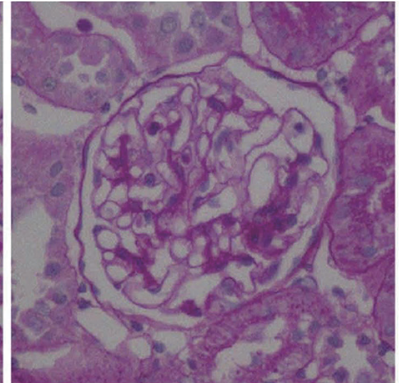

$\mathrm{STZ}+\mathrm{miR} 30 \mathrm{~s}$ mimic

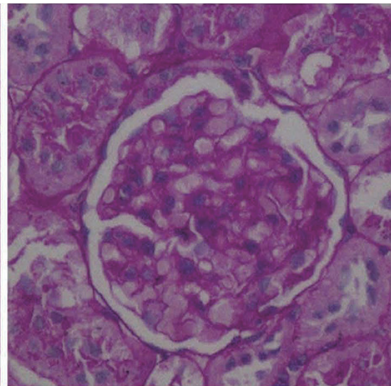

$\mathrm{STZ}+$ miR30s inhibitor
B

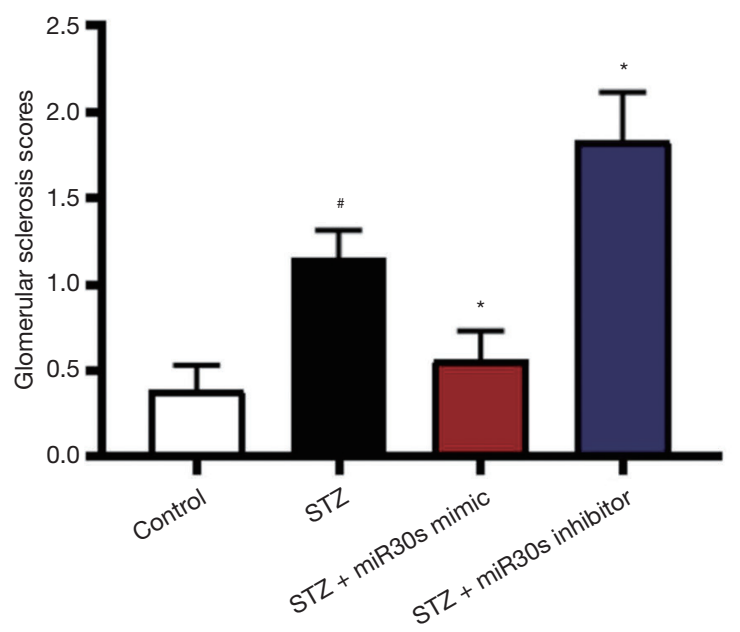

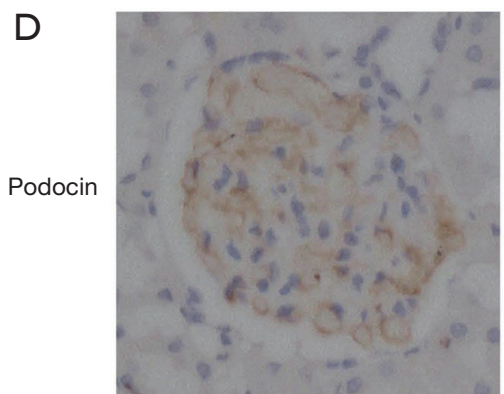

Control

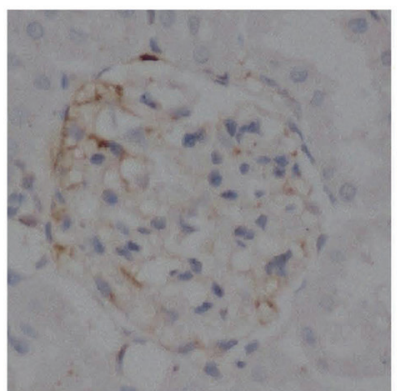

STZ
C

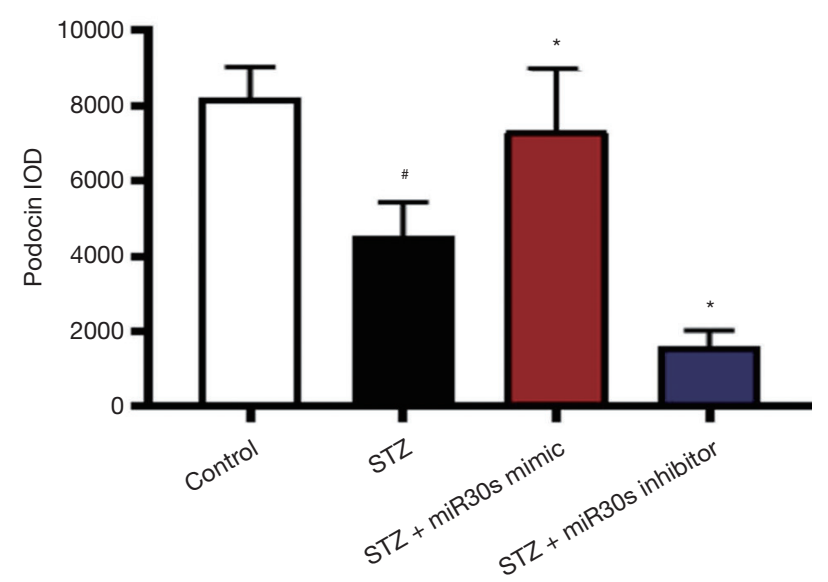

Figure 5 MicroRNA-30s modulate podocyte injury in vivo. Rats were divided into four groups: control, STZ, STZ + microRNA-30 family mimics, and STZ + microRNA-30 family inhibitors. (A) Representative photomicrographs (original magnification, $\times 400$ ) prepared from PAS-stained kidney sections. (B) The glomerular sclerosis scores of renal tissues among the four groups. (C) Quantitative analyses of the IOD of podocin are shown in the bar graph, and the data are expressed as the mean \pm SEM of three experiments. (D) IHC analyses of podocin protein expression among the four groups $(\times 40)$. Representative images from 4 mice in each group are shown. ${ }^{\#}, \mathrm{P}<0.05 v s$. the control group. *, $\mathrm{P}<0.05 v$ s. the STZ group. STZ, streptozotocin; PAS, Periodic Acid-Schiff stain; IHC, immunohistochemistry.

MicroRNAs, a class of non-coding RNAs, can bind to the 3'-UTR of their target mRNAs to participate in epigenetic regulation of their downstream signaling molecules. Recent studies have revealed the potential involvement of different microRNAs in different molecular and signaling pathways leading to DN. 
Page 10 of 14

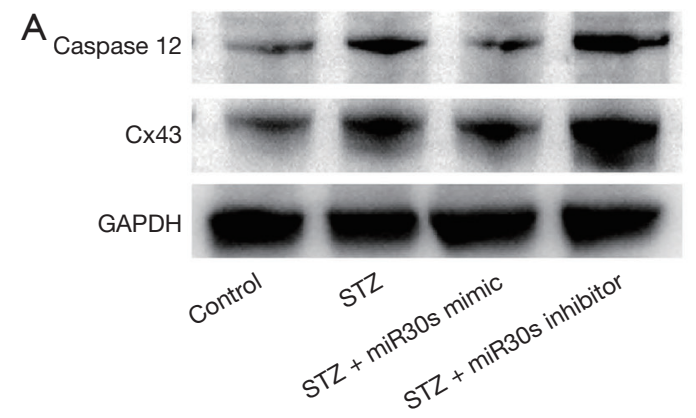

C

Caspase 12

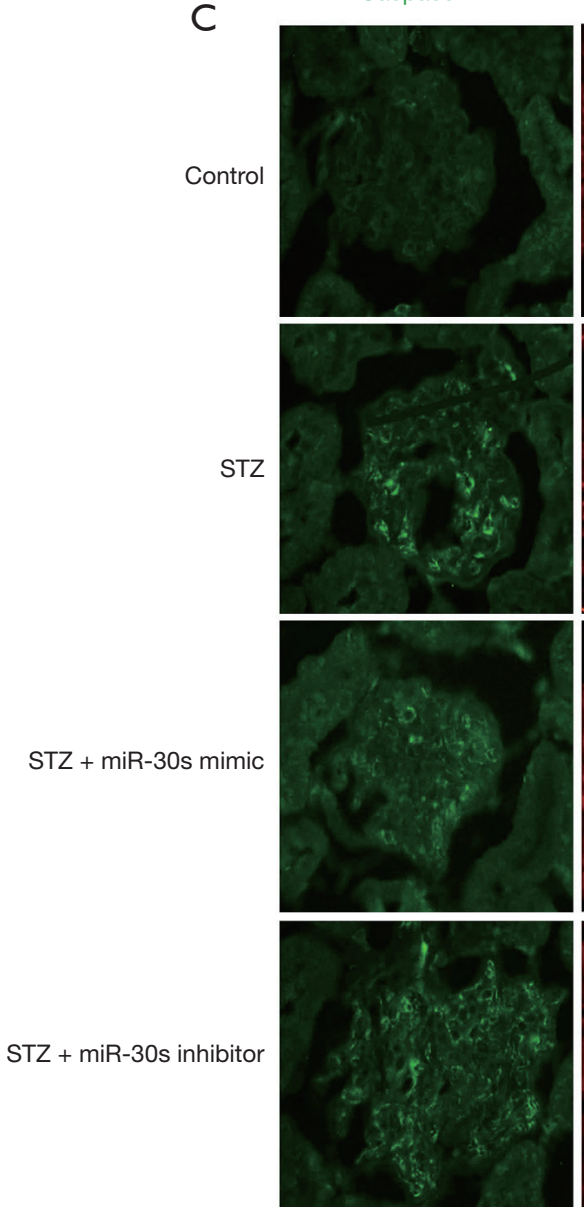

Li et al. The effect of microRNA-30/Cx43 axis on diabetic nephropathy

$\mathrm{B}$

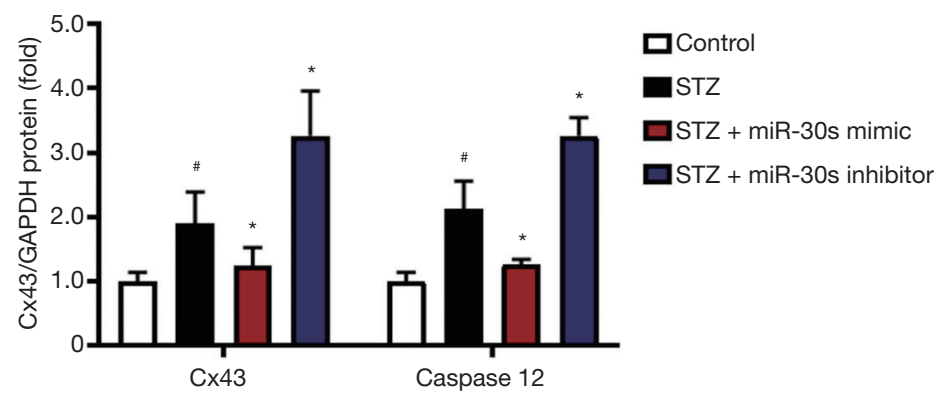

$\mathrm{Cx} 43$
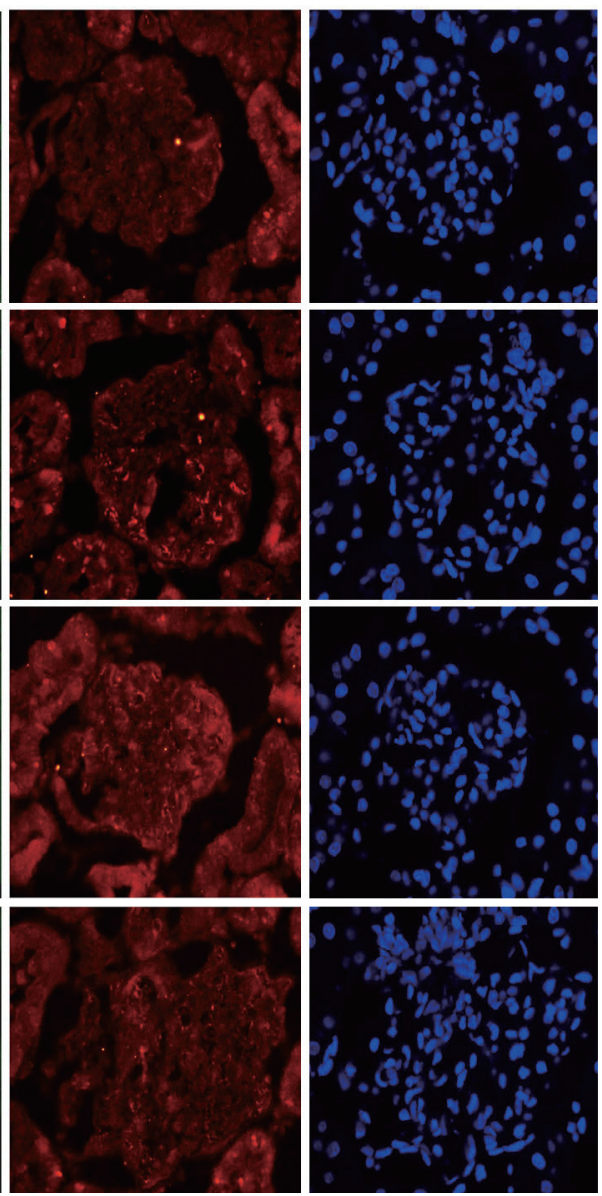

DAPI
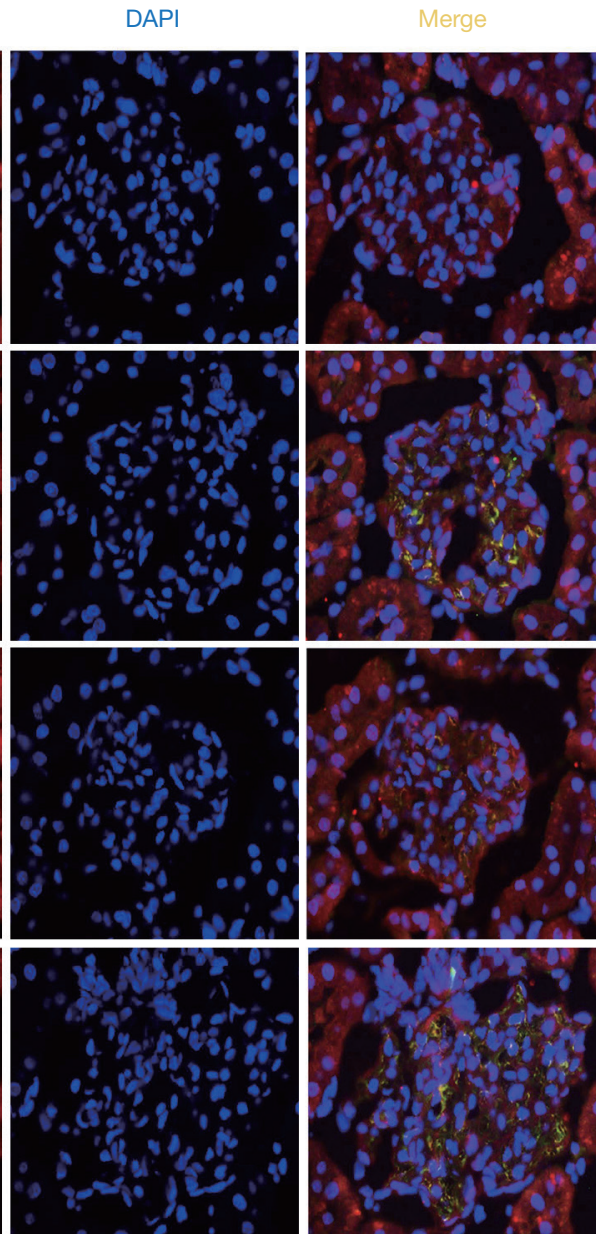

Figure 6 MicroRNA-30s modulate ERS through Cx43. Rats were divided into four groups: control, STZ, STZ + microRNA-30 family mimics, and STZ + microRNA-30 family inhibitors. (A,B) Western blot analyses of Cx43 and caspase12 protein levels. Quantitative ratios are shown as the relative optical densities of bands normalized to GAPDH expression. The data are representative of three similar experiments and quantified as the mean \pm SEM. ${ }^{*}, \mathrm{P}<0.05$ vs. the control group. *, $\mathrm{P}<0.05$ vs. the STZ group. (C) Indirect immunofluorescence staining of the expression and localization of caspase12 (green), Cx43 (red), and DAPI (blue; original magnification, $\times 400)$. STZ, streptozotocin; ERS, endoplasmic reticulum stress.

PI3K/Akt is a signaling pathway that is involved in the process of glomerular hypertrophy and podocyte injury in DN. Recent studies have revealed that microRNA- 216a, microRNA-217, microRNA-21, microRNA-200b, microRNA-200c and microRNA-192 have up-regulated the PI3K/Akt signaling pathway (26). 
Furthermore, microRNA-192, microRNA-215, microRNA-21 and microRNA-216a have been reported to up-regulate collagen expression in renal tissues and thereby promote the accumulation of extracellular matrix (ECM) proteins and induce glomerulosclerosis in DN (27-29), whereas microRNA-194, microRNA-23b, microRNA-146a and microRNA-326 have been identified as down-regulators of collagen expression and thereby have been recognized to be involved in the reduction of ECM protein accumulation and fibrosis in DN (30-32).

To date, there is no direct evidence regarding the regulation of B7-1 expression by microRNAs in renal cells in DN. However, several miRNAs expressed in renal tissues have been reported to regulate B7-1 expression in immune cells to participate in the pathogenesis of DN. For example, microRNA-125b-mediated up-regulation of B7-1 surface expression in macrophages can enhance macrophage activation, which is associated with the progression of diabetic nephropathy through podocyte damage and the inhibition of nephrin and podocin (33). Moreover, microRNA-155, microRNA-181d and microRNA-214mediated B7-1 up-regulation further promotes activation of inflammatory cytokine and thereby act as DNinducing miRNAs $(34,35)$. Nevertheless, microRNA-146a, microRNA-23b, microRNA-223 and microRNA-487b have been identified as down-regulators of B7-1 and act as DNinhibiting miRNAs (26).

In addition, Dai et al. have revealed that microRNA193a promotes the expression of ubiquitin carboxyterminal hydrolase 1 (UCH-L1), as a podocyte injury-inducing in $\mathrm{DN}$ via the $\mathrm{Wnt} / \beta$-catenin pathway (36).

As inhibition of dipeptidyl-peptidase-4 (DPP-4) has been reported to be a potential therapeutic intervention in $\mathrm{DN}$, microRNA-29s and Let-7s, inhibitors of DPP-4 gene, have been reported to be involved in attenuating DN $(37,38)$. MicroRNA-302a, microRNA-302c, microRNA-204 and microRNA-338 can down-regulate the expression of glucagon-like peptide-1 receptor (GLP1R), a receptor of a key substrate for DPP-4, which are also involved in the regulation of $\mathrm{DN}(39,40)$.

Earlier studies have confirmed that transforming growth factor beta (TGF- $\beta 1$ ) is a major cytokine responsible for glomerular hypertrophy and ECM accumulation in the mesangium. Thus, microRNA-192, microRNA216a, microRNA-21 and microRNA-195 have enhanced TGF- $\beta 1$ expression to elicit the progression of DN. In contrast, microRNA-141, microRNA-200a and microRNA$30 \mathrm{c}$ have been identified as down-regulators of TGF- $\beta 1$ signaling and thereby act as DN-inhibiting miRNAs (26). In line with the results of earlier studies (5), our data also confirms microRNA-30s are associated with podocyte injury. MicroRNAs usually function through their target genes. Therefore, we focused on $\mathrm{Cx} 43$ as a target of the microRNA-30 family, which was found by TargetScan software (Figure 1B). We confirmed that the microRNA-30 family regulates cx43 expression in podocytes through the following three observations: (I) $\mathrm{Cx} 43$ expression was elevated when microRNA-30 family expression was reduced in the HG group (Figure 1D,E,F,G). (II) $\mathrm{Cx} 43$ was inhibited by the overexpression of the microRNA-30 family in podocytes (Figure 1D,E,F,G). (III) Analysis of the luciferase reporter carrying the Cx43 3'-UTR showed microRNA30 s directly interact with this sequence and that the putative microRNA-30 family binding sites are essential for the regulation by the microRNA-30s (Figure 1C).

Earlier studies have proved the increased expression of unfolded protein response genes is associated with how much both clinical and pathological involvement of the kidney (41). Following earlier findings, growing evidence shows that the induction of free fatty acid, palmitate, and protein overload may cause ERS-induced podocyte apoptosis (42-44). ERS is induced during DN development, and the ERS pathway mediates apoptosis; this is hypothesized to be involved in podocyte injury (45). Also, Fujii et al. pointed out that ERS in podocytes may cause alterations in nephrin $\mathrm{N}$-glycosylation (46). It is accepted caspase 12 is a pro-apoptotic molecule specifically located in the outer membrane of the ER and is associated only with the mechanism of ERS-mediated apoptosis. In the present study, we confirmed podocyte apoptosis and caspase12 expression were upregulated, while the expression of podocin, a podocyte-specific protein, was downregulated in STZ-induced DN rats and HG-stimulated podocytes (Figures 2,3,5,6). These findings suggest ERS-mediated apoptosis is associated with podocyte injury in $\mathrm{DN}$ both in vivo and in vitro.

Recently, growing evidence has showed that ERS may be associated with $\mathrm{Cx} 43$ in some diseases, and changes in ERS and GJs coexist in many pathologic conditions, including cancer and ischemic heart disease (47-49). Our in vitro and in vivo data prove the expression of caspase 12 is downregulated by microRNA-30 family mimics and Cx43 siRNA (Figures 2,3,6). The inhibition of microRNA30 s induced increased expression of caspase12, reduced expression of podocin, and increased podocyte apoptosis; these results could be reversed by silencing $\mathrm{Cx} 43$ expression 
(Figure 3). Double-immunofluorescence staining assays proved the co-localization of caspase 12 and $\mathrm{Cx} 43$ (Figure 6). These results further show the overexpression of the microRNA-30 family alleviates ERS by inhibiting $\mathrm{Cx} 43$ expression from reducing podocyte injury.

Despite the added precautions, there are limitations to the current study. First, there are too few methods and indicators to study MicroRNA-30 and Cx43 attenuating the apoptosis and damage of podocytes in vitro, as well as to study the endoplasmic reticulum stress in podocyte damage in diabetic nephropathy. Second, renal pathology and podocyte ultrastructure changes under the electron microscope are not observed. Third, the biochemical indicators of the rats such as fasting blood glucose, cholesterol, triacylglycerol, low density lipoprotein, high density lipoprotein, blood creatinine, endogenous creatinine clearance, 24-hour urine albumin and its excretion rate and so on are not evaluated. There is no doubt that adding the above indicators would make the results more reliable.

In conclusion, our study supplies evidence the overexpression of microRNA-30 family members prevents HG-induced podocyte injury and attenuates ERS by modulating $\mathrm{Cx} 43$. These data suggest the microRNA-30/ $\mathrm{Cx} 43 /$ ERS axis may be a potential therapeutic target for DN patients.

\section{Acknowledgments}

Funding: Grants supported this work from the project of Jiangsu provincial Health and Family Planning Commission to MY (No. KY2018105), the Changzhou Health Commission youth talent Science and Technology project to ML (QN201907).

\section{Footnote}

Reporting Checklist: The authors have completed the ARRIVE reporting checklist. Available at http://dx.doi. org/10.21037/atm-20-6989

Data Sharing Statement: Available at http://dx.doi. org/10.21037/atm-20-6989

Conflicts of Interest: All authors have completed the ICMJE uniform disclosure form (available at http://dx.doi. org/10.21037/atm-20-6989). Dr. MY reports grants from the Project of Jiangsu Provincial Health and Family Planning Commission, during the conduct of the study;
Dr. ML reports grants from the Changzhou Health Commission youth talent Science and Technology project, during the conduct of the study. FH serves as an unpaid Associate Editor-in-Chief of Annals of Translational Medicine from Mar 2020 to Mar 2021. The other authors have no conflicts of interest to declare.

Ethical Statement: The authors are accountable for all aspects of the work in ensuring that questions related to the accuracy or integrity of any part of the work are appropriately investigated and resolved. All animal experiments were performed in accordance with the guidelines for animal care and approved by the Institutional Animal Care and Use Committee at Soochow University, China (No. 2020-013).

Open Access Statement: This is an Open Access article distributed in accordance with the Creative Commons Attribution-NonCommercial-NoDerivs 4.0 International License (CC BY-NC-ND 4.0), which permits the noncommercial replication and distribution of the article with the strict proviso that no changes or edits are made and the original work is properly cited (including links to both the formal publication through the relevant DOI and the license). See: https://creativecommons.org/licenses/by-nc-nd/4.0/.

\section{References}

1. Conserva F, Pontrelli P, Accetturo M, et al. The pathogenesis of diabetic nephropathy: focus on microRNAs and proteomics. J Nephrol 2013;26:811-20.

2. Najafian B, Alpers CE, Fogo AB. Pathology of human diabetic nephropathy. Contrib Nephrol 2011;170:36-47.

3. Zhou L, Liu Y. Wnt/beta-catenin signalling and podocyte dysfunction in proteinuric kidney disease. Nat Rev Nephrol 2015;11:535-45.

4. Susztak K, Raff AC, Schiffer M, et al. Glucose-induced reactive oxygen species cause apoptosis of podocytes and podocyte depletion at the onset of diabetic nephropathy. Diabetes 2006;55:225-33.

5. Wu J, Zheng C, Fan Y, et al. Downregulation of microRNA-30 facilitates podocyte injury and is prevented by glucocorticoids. J Am Soc Nephrol 2014;25:92-104.

6. Yao J, Liang L, Huang S, et al. MicroRNA-30d promotes tumor invasion and metastasis by targeting Galphai2 in hepatocellular carcinoma. Hepatology 2010;51:846-56.

7. Harvey SJ, Jarad G, Cunningham J, et al. Podocyte-specific deletion of dicer alters cytoskeletal dynamics and causes 
glomerular disease. J Am Soc Nephrol 2008;19:2150-8.

8. Zhao Y, Wu J, Zhang M, et al. Angiotensin II induces calcium/calcineurin signaling and podocyte injury by downregulating microRNA-30 family members. J Mol Med (Berl) 2017;95:887-98.

9. Liu WT, Peng FF, Li HY, et al. Metadherin facilitates podocyte apoptosis in diabetic nephropathy. Cell Death Dis 2016;7:2477.

10. Peng R, Zhou L, Zhou Y, et al. MiR-30a Inhibits the Epithelial--Mesenchymal Transition of Podocytes through Downregulation of NFATc3. Int J Mol Sci 2015;16:24032-47.

11. Zhou Z, Chen Y, Zhang D, et al. MicroRNA-30-3p Suppresses Inflammatory Factor-Induced Endothelial Cell Injury by Targeting TCF21. Mediators Inflamm 2019;2019:1342190.

12. Bridge G, Monteiro R, Henderson S, et al. The microRNA-30 family targets DLL4 to modulate endothelial cell behavior during angiogenesis. Blood 2012;120:5063-72.

13. Liu YF, Spinelli A, Sun LY, et al. MicroRNA-30 inhibits neointimal hyperplasia by targeting $\mathrm{Ca}(2+) /$ calmodulindependent protein kinase II $\delta$ (CaMKII $\delta$ ). Sci Rep 2016;6:26166.

14. Kumar NM, Gilula NB. The gap junction communication channel. Cell 1996;84:381-8.

15. Yang M, Wang B, Li M, et al. Connexin 43 is involved in aldosterone-induced podocyte injury. Cell Physiol Biochem 2014;34:1652-62.

16. Yaoita E, Yao J, Yoshida Y, et al. Up-regulation of connexin43 in glomerular podocytes in response to injury. Am J Pathol 2002;161:1597-606.

17. Yan Q, Gao K, Chi Y, et al. NADPH oxidase-mediated upregulation of connexin43 contributes to podocyte injury. Free Radic Biol Med 2012;53:1286-97.

18. Huang T, Wan Y, Zhu Y, et al. Downregulation of gap junction expression and function by endoplasmic reticulum stress. J Cell Biochem 2009;107:973-83.

19. Szegezdi E, Logue SE, Gorman AM, et al. Mediators of endoplasmic reticulum stress-induced apoptosis. EMBO Rep 2006;7:880-5.

20. García de la Cadena S, Massieu L. Caspases and their role in inflammation and ischemic neuronal death. Focus on caspase-12. Apoptosis 2016;21:763-77.

21. Yoneda T, Imaizumi K, Oono K, et al. Activation of caspase-12, an endoplastic reticulum (ER) resident caspase, through tumor necrosis factor receptor-associated factor 2-dependent mechanism in response to the ER stress. J
Biol Chem 2001;276:13935-40.

22. Fineberg D, Jandeleit-Dahm KA, Cooper ME. Diabetic nephropathy: diagnosis and treatment. Nat Rev Endocrinol 2013;9:713-23.

23. Du P, Fan B, Han H, et al. NOD2 promotes renal injury by exacerbating inflammation and podocyte insulin resistance in diabetic nephropathy. Kidney Int 2013;84:265-76.

24. Tesch GH, Allen TJ. Rodent models of streptozotocininduced diabetic nephropathy. Nephrology (Carlton) 2007;12:261-6.

25. Lenoir O, Jasiek M, Henique C, et al. Endothelial cell and podocyte autophagy synergistically protect from diabetesinduced glomerulosclerosis. Autophagy 2015;11:1130-45.

26. Dewanjee S, Bhattacharjee N. MicroRNA: A new generation therapeutic target in diabetic nephropathy. Biochem Pharmacol 2018;155:32-47.

27. Wu H, Kong L, Zhou S, et al. The role of microRNAs in diabetic nephropathy. J Diabetes Res 2014;2014:920134.

28. Kato M, Wang L, Putta S, et al. Post-transcriptional up-regulation of Tsc-22 by Ybx1, a target of miR-216a, mediates TGF-\{beta\}-induced collagen expression in kidney cells. J Biol Chem 2010;285:34004-15.

29. Kölling M, Kaucsar T, Schauerte C, et al. Therapeutic miR-21 Silencing Ameliorates Diabetic Kidney Disease in Mice. Mol Ther 2017;25:165-80.

30. John A, Kundu S, Pushpakumar S, et al. GYY4137, a Hydrogen Sulfide Donor Modulates miR194-Dependent Collagen Realignment in Diabetic Kidney. Sci Rep 2017;7:10924.

31. Zhao B, Li H, Liu J, et al. MicroRNA-23b Targets Ras GTPase-Activating Protein SH3 Domain-Binding Protein 2 to Alleviate Fibrosis and Albuminuria in Diabetic Nephropathy. J Am Soc Nephrol 2016;27:2597-608.

32. Morishita Y, Imai T, Yoshizawa H, et al. Delivery of microRNA-146a with polyethylenimine nanoparticles inhibits renal fibrosis in vivo. Int $\mathrm{J}$ Nanomedicine 2015;10:3475-88.

33. Chaudhuri AA, So AY, Sinha N, et al. MicroRNA$125 \mathrm{~b}$ potentiates macrophage activation. $\mathrm{J}$ Immunol 2011;187:5062-68.

34. Mbongue JC, Nieves HA, Torrez TW, et al. The Role of Dendritic Cell Maturation in the Induction of InsulinDependent Diabetes Mellitus. Front Immunol 2017;8:327.

35. Su XW, Lu G, Leung CK, et al. miR-181d regulates

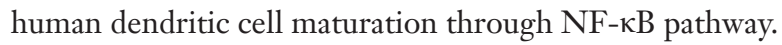
Cell Prolif 2017;50:e12358.

36. Dai H, Liu Q, Liu B. Research Progress on Mechanism of 
Podocyte Depletion in Diabetic Nephropathy. J Diabetes Res 2017;2017:2615286.

37. Shi S, Koya D, Kanasaki K. Dipeptidyl peptidase-4 and kidney fibrosis in diabetes. Fibrogenesis Tissue Repair 2016;9:1.

38. Kanasaki K. The role of renal dipeptidyl peptidase-4 in kidney disease: renal effects of dipeptidyl peptidase-4 inhibitors with a focus on linagliptin. Clin Sci (Lond) 2018;132:489-507.

39. Mao Y, Mohan R, Zhang S, et al. MicroRNAs as pharmacological targets in diabetes. Pharmacol Res 2013;75:37-47.

40. Jo S, Chen J, Xu G, et al. miR-204 Controls GlucagonLike Peptide 1 Receptor Expression and Agonist Function. Diabetes 2018;67:256-64.

41. Lindenmeyer MT, Rastaldi MP, Ikehata M, et al. Proteinuria and hyperglycemia induce endoplasmic reticulum stress. J Am Soc Nephrol 2008;19:2225-36.

42. Sieber J, Lindenmeyer MT, Kampe K, et al. Regulation of podocyte survival and endoplasmic reticulum stress by fatty acids. Am J Physiol Renal Physiol 2010;299:F821-9.

43. Inagi R, Nangaku M, Onogi H, et al. Involvement of endoplasmic reticulum (ER) stress in podocyte injury induced by excessive protein accumulation. Kidney Int 2005;68:2639-50.

44. Tao JL, Wen YB, Shi BY, et al. Endoplasmic reticulum

Cite this article as: $\mathrm{Li} M, \mathrm{Ni} W$, Zhang M, Liu S, Chen M, Hong X, Ma Y, Yu X, Wang W, Yang M, Hua F. MicroRNA-30/ $\mathrm{Cx} 43$ axis contributes to podocyte injury by regulating ER stress in diabetic nephropathy. Ann Transl Med 2020;8(24):1674. doi: 10.21037/atm-20-6989 stress is involved in podocyte apoptosis induced by saturated fatty acid palmitate. Chin Med J (Engl) 2012;125:3137-42.

45. Liu G, Sun Y, Li Z, et al. Apoptosis induced by endoplasmic reticulum stress involved in diabetic kidney disease. Biochem Biophys Res Commun 2008;370:651-6.

46. Fujii Y, Khoshnoodi J, Takenaka H, et al. The effect of dexamethasone on defective nephrin transport caused by ER stress: a potential mechanism for the therapeutic action of glucocorticoids in the acquired glomerular diseases. Kidney Int 2006;69:1350-9.

47. Kimata Y, Ishiwata-Kimata Y, Yamada S, et al. Yeast unfolded protein response pathway regulates expression of genes for anti-oxidative stress and for cell surface proteins. Genes Cells 2006;11:59-69.

48. Huang T, Zhu Y, Fang X, et al. Gap junctions sensitize cancer cells to proteasome inhibitor MG132-induced apoptosis. Cancer Sci 2010;101:713-21.

49. Rab A, Bartoszewski R, Jurkuvenaite A, et al. Endoplasmic reticulum stress and the unfolded protein response regulate genomic cystic fibrosis transmembrane conductance regulator expression. Am J Physiol Cell Physiol 2007;292:C756-66.

(English Language Editor: J. Chapnick) 